\title{
Inhibition of mitogen-activated protein kinase signaling pathway sensitizes breast cancer cells to endoplasmic reticulum stress-induced apoptosis
}

\author{
FEN YANG ${ }^{1,2^{*}}$, XIAO YAN TANG ${ }^{3 *}, \mathrm{HAO} \mathrm{LIU}^{4}$ and ZHI WEN JIANG ${ }^{4}$ \\ ${ }^{1}$ Department of Tumor Radiotherapy, the First Affiliated Hospital of Bengbu Medical College, Bengbu, Anhui; \\ ${ }^{2}$ Department of Biochemistry and Molecular Biology, School of Basic Medical Sciences, Nanjing Medical \\ University, Nanjing, Jiangsu; ${ }^{3}$ Department of Clinical Laboratory, Nanjing Chest Hospital, Nanjing, \\ Jiangsu; ${ }^{4}$ Department of Pharmacy, Bengbu Medical College, Bengbu, Anhui, P.R. China
}

Received November 5, 2015; Accepted December 17, 2015

DOI: $10.3892 /$ or.2016.4580

\begin{abstract}
Accumulation of unfolded proteins in the endoplasmic reticulum (ER) induces ER stress which is observed in many human diseases, including breast cancer. Cellular adaptation to ER stress is mediated by the unfolded protein response (UPR), which aims at restoring ER homeostasis. Higher levels of GRP78 expression indicates constitutive activation of the UPR in breast cancer leading to breast cancer cells that are relatively resistant to ER stress-induced apoptosis. Tunicamycin (TM), an ER stress inducer, constitutively activates the mitogen-activated protein kinase (MAPK)/extracellular signal regulated kinase (ERK), and (MEK)/ERK pathway which plays a role in upregulation of GRP78 by ER stress in that inhibition of MEK by U0126 reduces the levels of GRP78 and blocks its upregulation by TM. Inhibition of the MEK/ERK pathway by U0126 sensitizes breast cancer cells to TM-induced apoptosis. Inhibition of GRP78 by siRNA knockdown enhances TM- and U0126-induced apoptosis in breast cancer cells. This sensitization of breast cancer cells to TM-induced apoptosis by inhibition of MEK/ERK and GRP78 is caspase-dependent, at least in part, by activation of caspase-4. These results seem to indicate that GRP78 has potential as a chemotherapeutical target and have important implications for new treatment strategies in breast cancer by combination with agents that induce ER stress with inhibitors of the MEK/ERK pathway.
\end{abstract}

Correspondence to: Dr Fen Yang, Department of Biochemistry and Molecular Biology, School of Basic Medical Sciences, Nanjing Medical University, 140 Han Zhong Road, Nanjing, Jiangsu, P.R. China

E-mail: yangfen@njmu.edu.cn

*Contributed equally

Key words: unfolded protein response, MEK/ERK pathway, ER stress, GRP78, apoptosis

\section{Introduction}

Endoplasmic reticulum (ER) is an essential cellular compartment where new secretory proteins are folded and assembled to their maturation. When suffering various conditions such as nutrient deprivation, hypoxia, alterations in glycosylation status and disturbances of calcium flux, the steady state of the ER environment is disturbed and misfolded or unfolded proteins are accumulated and aggregated in ER lumen, failure of ER coping with the excessive proteins load leads to ER stress (1). To reduce damage from ER stress, cells trigger unfolded protein response (UPR), which signals transient attenuation of protein translation, degradation of unfolded and misfolded proteins and the induction of molecular chaperones (2). The upregulation of molecular chaperones in response to expression of dominant negative ubiquitin may contribute to degradation of abnormal proteins (3).

Three known transmembrane sensors of ER stress are double-stranded RNA-activated protein kinase-like ER kinase (PERK), inositol-requiring enzyme 1 (IRE1) and activating transcription factor 6 (ATF6) (4). Under normal conditions, the luminal domains of these sensors are occupied by the ER chaperon glucose-regulated protein 78 (GRP78) (5). Upon ER stress, unfolded or misfolded proteins compete with these sensors for binding GRP78 segregating GRP78 from those of luminal domains. As a result, these sensors are activated by inducing phosphorylation and homodimerization of IRE1 and PERK, and relocalization of ATF6 to the Golgi $(6,7)$.

GRP78, is also known as immunoglobulin heavy chain binding protein (BIP), is a central regulator of ER function, an ER molecular chaperon, facilitating new protein folding and assembling, targeting misfolded proteins for degradation, binding $\mathrm{ER} \mathrm{Ca}{ }^{2+}$ and controlling the activation of transmembrane ER stress sensors $(8,9)$. Perhaps due to nutrient deprivation and hypoxia, increasing evidence shows that the UPR is indispensable in various solid tumors, investigators have reported elevated expression of UPR targets, such as GRP78 and GRP94 $(10,11)$. The expression of these genes and other components of UPR are also correlated with increased malignancy. It seems that some cancer cells may 
have adapted to ER stress by activation of UPR without resulting in apoptosis (12).

The UPR is a cytoprotective response to ER stress, but excessive or prolonged UPR results in apoptotic cell death. Many caspase family members participate in the process of ER stress-induced apoptosis, such as caspase-2, -3, -4, -7, -8 -9 and -12 (13-17). Among them, caspase-12 is thought to be a key mediator in rodents. However, caspase- 4 has been shown to play an important role in ER stress-induced apoptosis of human neuroblastoma and HeLa cells. Mutual action between caspase-4 and GRP78 suggests that caspase-4 lies in ER lumen $(18,19)$, this provides structural basis for regulation of ER stress-induced apoptosis by caspase- 4 .

Previous studies have shown that survival signaling pathways, such as the phosphatidylinositol 3-kinase (PI3K)/AKT and mitogen-activated protein kinase (MAPK)/extracellular signal regulated kinase (ERK) and (MEK)/ERK pathways, may also play roles in counteracting the apoptosis-inducing potential of ER stress. Particularly, constitutive activation of the MEK/ERK pathway is reported as a common cause for resistance of melanoma cells to apoptosis mediated by the death receptor and mitochondrial apoptotic pathways (20-23).

In the present study, we found that UPR was constitutively activated in the breast cancer cells compared to normal breast epithelial cells in which GRP78 expression of cancer cells was obviously higher than epithelial cells. Moreover, we found that tunicamycin (TM), an ER stress inducer, not only strongly activated UPR, but also the MEK/ERK pathway, and inhibition of the pathway sensitized breast cancer cells to TM-induced apoptotic cell death due to downregulation of GRP78 expression. This sensitization of breast cancer cells to TM-induced apoptosis by inhibition of MEK/ERK and GRP78 was caspase-dependent, at least in part, by activation of caspase-4. These results indicated that GRP78 is a possible chemotherapeutical target and have important implications for new treatment strategies in breast cancer by combination of agents that induce ER stress with inhibitors of the MEK/ERK pathway.

\section{Materials and methods}

Cell lines. Human breast cancer cell lines MCF-7, MDA-MB-231 and SK-BR-3 were cultured in Department of Biochemistry and Molecular Biology, Nanjing Medical University. Human mammary epithelial cell line MCF-10A and human pneumonic epithelial cell WI-38 was purchased from the cell bank of Academia Sinica. They were cultured at $37^{\circ} \mathrm{C}$ in the presence of $5 \% \mathrm{CO}_{2}$ in Dulbecco's Modified Eagle's medium (DMEM; Gibco) supplemented with $10 \%$ fetal calf serum (FCS) and $100 \mathrm{U} / \mathrm{ml}$ penicillin $\mathrm{G}$ and $100 \mathrm{mg} / \mathrm{ml}$ streptomycin.

Clinical specimens. Clinical specimens were collected from the patients registered at the First Affiliated Hospital of Bengbu Medical College (Bengbu, China) between 2005 and 2008 with the patients consent and Ethics Committee approval. To monitor GRP78 protein immunostaining with clinicopathological stages, patient medical records were retrospectively reviewed at the time when the study sample was obtained. All samples were randomly selected and arranged as three groups: breast cancers, adjacent non-cancerous tissue and breast fibroadenoma.

Antibodies, recombinant proteins and other reagents. The Vectastain ABC kit and the DAB kit were from Wuhan Boster Biotechnology Co. Tunicamycin (TM) was purchased from Sigma Chemical Co. (Castle Hill, Australia), and dissolved in dimethyl sulfoxide (DMSO) and made to stock solutions of $1 \mathrm{mmol} / \mathrm{l}$. The rabbit polyclonal antibody against GRP78, ERK1 and ERK2 were purchased from Santa Cruz Biotechnology Co. The mouse monoclonal antibody against P-ERK1/2 and $\beta$-actin were purchased from Santa Cruz Biotechnology Co. The mouse IgG and rabbit IgG were purchased from Sigma Chemical Co. Propidium iodide (PI) was purchased from Sigma Chemical Co. Reverse transcription-qPCR assay kit was from Takara Co. TRIzol was from Invitrogen Co. The siGENOME SMART pool reagents, the siGENOME SMART pool GRP78 (M-008198-01) and control non-targeting siRNA pool (D-001206-13-20) were obtained from Dharmacon. Opti-MEM medium and Lipofectamine 2000 reagent were purchased from Invitrogen Co.

Immunohistochemical staining. Five micrometer-thick sections were cut from the formalin-fixed, paraffin-embedded block of each case. Sections were deparaffinized in xylene and rehydrated through graded decreasing concentrations of alcohol. Antigen was repaired in $0.01 \mathrm{~mol} / 1$ of citrate buffer ( $\mathrm{pH}$ 6.0) by heating in a microwave oven for $5 \mathrm{~min}$ and repeating three times. Rabbit anti-human GRP78 antibody was added at a dilution of 1:100 in phosphate-buffered saline (PBS) for $1 \mathrm{~h}$ at $37^{\circ} \mathrm{C}$. The Vectastain $\mathrm{ABC}$ kit was used to bind the antibodies according to the manufacturer's instructions and the binding sites were visualized using the DAB kit. The sections were counterstained with Harris hematoxylin. Negative controls were performed by omission of the primary antibody replaced by PBS in each experiment and each heavy pigmented case.

Each section was observed in $10 \mathrm{HP}$ visual fields (VFs), and 200 cells/VF were counted. Score of positive cells $<10 \%$ was regarded as $0,10-50 \%$ as $1,51-75 \%$ as 2 , and $\geq 75 \%$ as 3 . No staining was 0 , weakly positive staining was 1 , moderately positive staining was 2 and strongly positive staining was 3 . An immunoreactive score (IRS) was derived by multiplying the score of percentage of positive cells with the score of staining intensity. IRS $=0$ was regarded as negative; IRS=4 was weakly positive; and IRS $\geq 4$ was strongly positive.

PI uptake assay and apoptosis. Cells were seeded at $1 \times 10^{5} /$ well into 24-well plates and allowed to reach exponential growth for 16-24 h before treatment. PI staining procedure followed the manufacturer's protocol. PI buffer was mixed with $100 \mathrm{ml}$ $\mathrm{dH}_{2} \mathrm{O}, 0.005 \mathrm{~g}$ PI, $0.1 \mathrm{~g}$ trisodium citrate, $100 \mu \mathrm{l}$ Triton X-100, covering with foil and keeping at $4^{\circ} \mathrm{C}$. Quantitation of apoptotic cells were represented by measurement of sub-G1 DNA content using PI staining in FCM.

Protein extraction and western blot analysis. Whole cell extracts were obtained by lysing cells in a Triton X-100based lysis buffer $(10 \mathrm{mmol} / 1 \mathrm{Tris}-\mathrm{HCl} \mathrm{pH} 7.4,140 \mathrm{~mol} / 1$ $\mathrm{NaCl}, 0.5 \mathrm{mmol} / 1 \mathrm{CaCl}_{2}, 10.5 \mathrm{mmol} / 1 \mathrm{MgCl}_{2}, 3 \mathrm{mmol} / 1 \mathrm{NaN}_{3}$, 
Triton X-100 $2 \mathrm{ml}$, adding to $12 \mu \mathrm{mol} / 1$ leupeptin, $1 \mathrm{mmol} / \mathrm{l}$ PMSF, respectively, before use. Extraction for phosphorylated proteins needs adding to $50 \mathrm{mmol} / 1 \mathrm{NaF}$ and $1 \mathrm{mmol} / \mathrm{l}$ $\mathrm{Na}_{3} \mathrm{VO}_{4}$ ). The protein content of cell extracts was determined by the BCA assay. A total load of 30-40 $\mu \mathrm{g}$ proteins was electrophoresed on $10 \%$ SDS-PAGE gels and transferred to PVDF membranes. Membranes were blocked, incubated with primary antibody at the appropriate concentration (1:500-1,000), and subsequently incubated with horseradish peroxidase-conjugated goat anti-rabbit IgG or goat antimouse IgG (1:5,000-10,000 dilution). Labeled bands were detected by Renaissance Western Blot Chemiluminescence Reagent (Pierce Co.) and exposed on Hyper MP autoradiography film (Amersham). The densities of the bands were quantitated by Gel Imaging System as proteins relatively expression levels.

Reverse transcription and quantitative PCR (RT-qPCR). Total RNA was extracted according to the protocol and RNA concentrations were determined spectrophotometrically at $260 \mathrm{~nm}$. Total RNA $(1 \mu \mathrm{g})$ was reverse transcribed to cDNA in a total volume of $20 \mu \mathrm{l}$ system using a RT reaction kit (Takara). Quantitative PCR was performed using an Mx3000P RealTime PCR system (Applied Biosystems) according to the manufacturer's instructions and SYBR Premix Ex Taq (Takara) as a DNA-specific fluorescent dye. PCR was carried out for 40 cycles of $95^{\circ} \mathrm{C}$ for $10 \mathrm{sec}$ and $60^{\circ} \mathrm{C}$ for $1 \mathrm{~min}$. All the reactions were repeated three times. Gene expression levels were calculated relative to GAPDH as a $2^{-\Delta \Delta \mathrm{Ct}}$ value using Stratagene Mx3000P software. Ratio of the treatment group $2^{-\Delta \Delta \mathrm{Ct}}$ value and the negative group represents relative expression levels. The primers used for amplification were as follows: GRP78, 5'-GTTTGCTGAGGAAGACAAAAAGCTC-3' and 5'-CACTTCCATAGAGTTTGCTGATAATTG-3'; GAPDH, 5'-GGGAAGGTGAAGGTCGGAGTC-3' and 5'-AGCAGAG GGGGCAGAGATGAT-3'. Primers and probes for GRP78 and GAPDH were from Sangon Co., Shanghai, China.

Small RNA interference (RNAi) transfection. Breast cancer cells MCF-7 and MDA-MB-231 were seeded at $5 \times 10^{4}$ cells/well in 24-well plates and allowed to reach $\sim 50 \%$ confluence on the day of transfection. Cells were transfected with 50-100 nmol/l siRNA in Opti-MEM medium with $10 \%$ FCS using Lipofectamine 2000 reagent according to the manufacturer's transfection protocol. Twenty-four hours after transfection, the cells were switched into medium containing $10 \%$ FCS and treated as designed before quantitation of apoptotic cells by measurement of sub-G1 DNA content using the PI method in FCM. Efficiency of siRNA transfection was measured by western blot analysis $24 \mathrm{~h}$ later.

Caspase-4 activity assay. Caspase-4 can catalyze substrate acetyl-Leu-Val-Asp $p$-nitroanilide (Ac-LEVD- $p$ NA) to flavous $p$-nitroaniline ( $p$-NA) with strong absorbance at the wavelength of $405 \mathrm{~nm}$, thus, absorbance was measured to determine caspase-4 activity. Total proteins were extracted as previously described in western blot analysis and quantitated by Bradford method. Measurement of caspase- 4 activity was carried out at the wavelength of $405 \mathrm{~nm}$, according to the manufacturer's protocol.
Table I. Expression of GRP78 in tissue by immunohistochemistry.

\begin{tabular}{lccrc}
\hline & & \multicolumn{3}{c}{ GRP78 (IRS) } \\
\cline { 3 - 5 } Specimen & Cases & $0(\%)$ & $1-4(\%)$ & $\geq 4(\%)$ \\
\hline Breast fibroadenoma & 20 & $16(80)$ & $4(20)$ & 0 \\
Cancer adjacent tissue & 50 & $10(20)$ & $35(70)$ & $5(10)$ \\
Breast cancer tissue & 50 & $1(2)$ & $7(14)$ & $42(84)$
\end{tabular}

GRP78 expression was detected in 20 cases of breast fibroadenoma, 50 cases of breast cancer and 50 cases of cancer adjacent tissue. IRS, 0 was regarded as negative; IRS, 1-4 was weakly positive; and IRS $\geq 4$ was strongly positive. IRS, immunoreactive score.

Statistical analysis. The statistical software SPSS, version 16.0, and the Student's t-test were used for statistical analysis. A P-value of $<0.05$ was considered to indicate a statistical significance.

\section{Results}

GRP78 expression is increased in breast cancer. In cell lines, GRP78 expression of breast cancer was significantly higher than that of normal epidermic cells both in protein and mRNA levels (Fig. 1A and B). GRP78 expression in paraffin blocks of breast cancer tissues using immunohistochemical staining is shown in Fig. 1C, the tissues were from 50 cases of breast cancer, 50 cases of adjacent non-cancerous tissue and 20 cases of breast fibroadenoma. GRP78 was expressed at significantly higher levels in breast cancer tissues in comparison with that in adjacent non-cancerous tissue and breast fibroadenoma $(\mathrm{P}<0.01$, Dunnette t-test), GRP78 was strongly positive in breast cancer (84\%), weakly positive in adjacent non-cancerous tissue $(70 \%)$, and markedly negative in breast fibroadenoma (80\%) (Table I). These results indicated that UPR was constitutively activated in breast cancer.

Breast cancer cells are relatively resistant to ER stress-induced apoptosis. We treated MCF-7, MDA-MB-231 cells with TM, an ER stress inducer. TM markedly upregulated GRP78 expression and in $24 \mathrm{~h}$ the upregulation peaked (Fig. 2A), indicative of further activation of the UPR. At the same time, we treated MCF-7, MDA-MB-231, MCF-10A and WI-38 cells with TM, respectively, at a range of concentrations for $48 \mathrm{~h}$ to study the apoptosis induction potential of ER stress. However, the two breast cancer cell lines were induced from minimal to moderate levels of apoptosis ( $<30 \%$ apoptotic cells), even when used at relatively high concentration (TM at $12 \mu \mathrm{mol} / \mathrm{l}$ ), which efficiently killed MCF-10A and WI-38 by induction of apoptosis (Fig. 2B).

Inhibition of MEK/ERK sensitizes breast cancer cells to ER stress-induced apoptosis. Constitutive activation of the $\mathrm{MEK} / \mathrm{ERK}$ pathway is reported as a commonly acceptable reason for resistance of cancer cells to apoptosis. Therefore, we monitored if ER stress induced activation of the MEK/ERK 

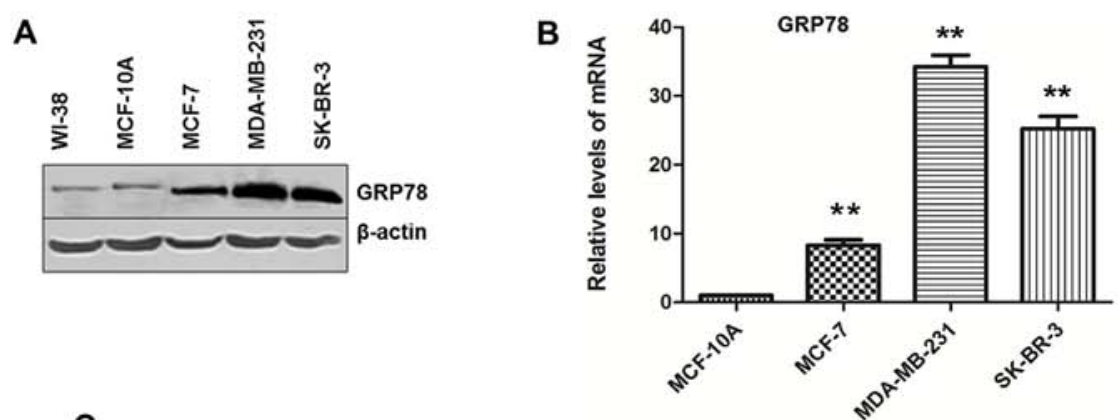

C

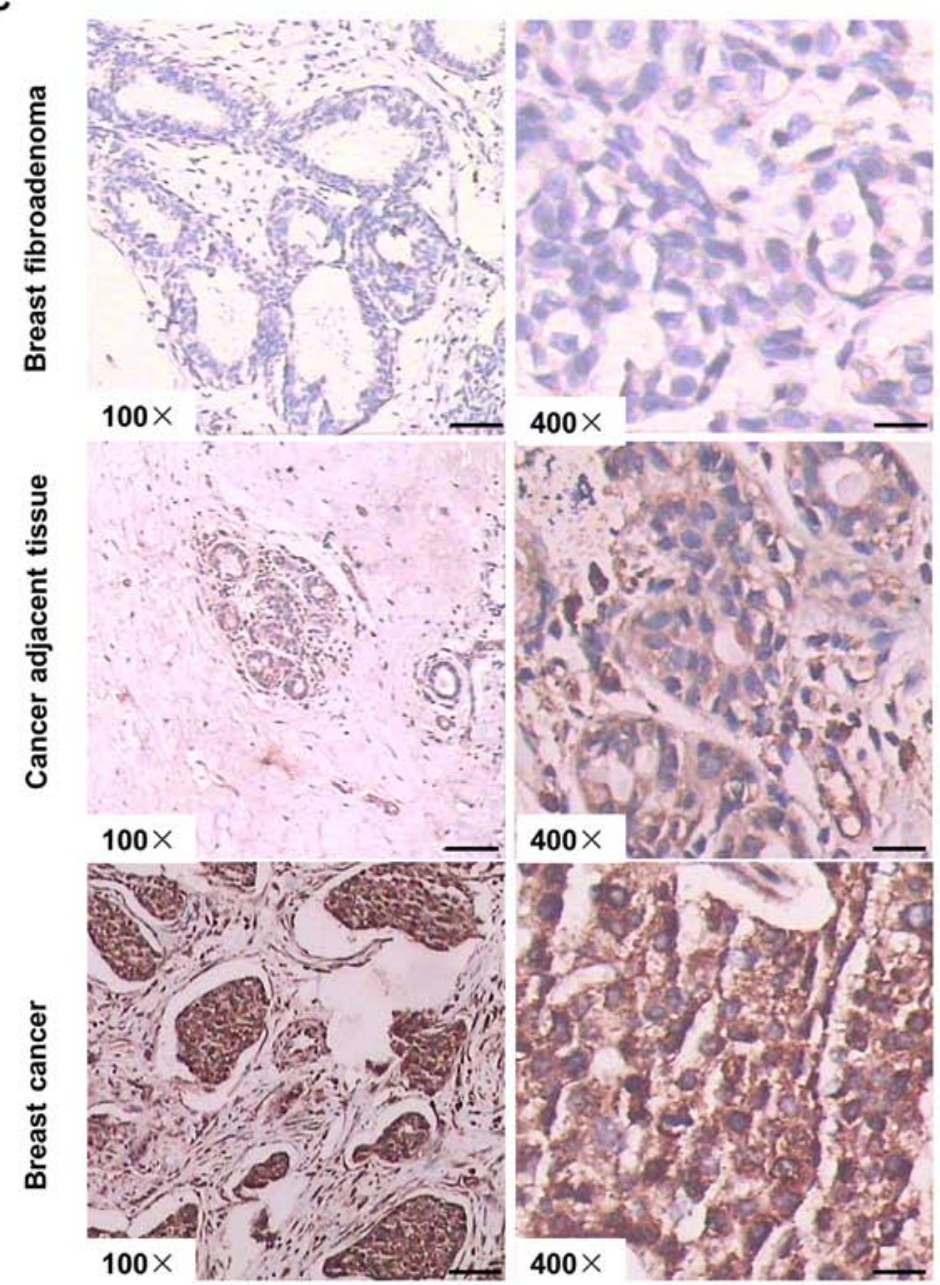

Figure 1. GRP78 was strongly positive in breast cancer. (A) GRP78 protein expression was strongly positive in breast cancer cell lines MCF-7, MDA-MB-231 and SK-BR-3 compared to normal cell lines MCF-10A and WI-38. (B) GRP78 mRNA expression in breast cancer cell lines was markedly higher than breast normal cell lines. ${ }^{* *} \mathrm{P}<0.01$ for breast cancer cell lines MCF-7, MDA-MB-231 and SK-BR-3 compared to normal cell lines MCF-10A, respectively. (C) GRP78 expression was strongly positive in breast cancer tissue, weakly positive in adjacent non-cancerous tissue and negative in breast fibroadenoma. Images were captured at a magnification of $\mathrm{x} 100$ and $\mathrm{x} 400$. Scale bars, $100 \mu \mathrm{m}$ in images at a magnification of $\mathrm{x} 100$, and $25 \mu \mathrm{m}$ in images at a magnification of $\mathrm{x} 400$.

pathway by examining phosphorylation (activation) of ERK1/2 in whole cell lysates from MCF-7 and MDA-MB-231 cells with exposure to TM. As shown in Fig. 3A, ERK1/2 was weakly activated in control cell lines, but the levels of activation were constitutively increased by treatment with TM, indicating that ER stress induced further ERK1/2 activation in breast cancer cells. We next measured the effect of MEK inhibition on TM-induced apoptosis in breast cancer cells by treating MCF-7 and MDA-MB-231 cells with the MEK inhibitor U0126 $1 \mathrm{~h}$ before TM treatment for another $24 \mathrm{~h}$. As shown in Fig. 3B and C, not only U0126 inhibited activation of ERK1/2 in the presence or absence of TM, but it significantly sensitized the cells to TM-induced apoptosis $(\mathrm{P}<0.01$, Student's t-test). However, the MEK inhibitor alone did not cause appreciable apoptotic cell death in the cell lines.

U0126 downregulates GRP78 expression in both protein and $m R N A$ levels. GRP78 is believed to play an essential role in protection of cells from ER stress-induced apoptosis (24). Therefore, we deduced that sensitization of breast cancer cells to ER stress-induced apoptosis by inhibition of MEK/ERK probably related to regulation of GRP78. As shown in Fig. 4A 
A

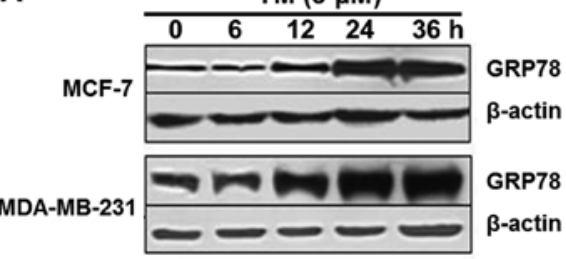

B

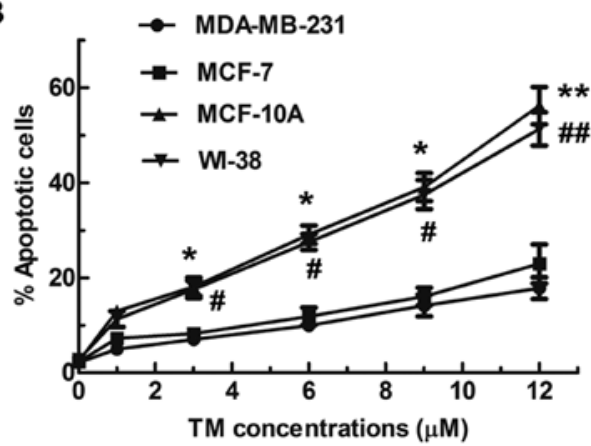

Figure 2. Breast cancer cell lines were relatively resistant to ER stress-induced apoptosis. (A) Tunicamycin (TM) induced activation of the UPR in the breast cancer cells. Whole-cell lysates from MCF-7 and MDA-MB-231 cells with or without treatment with TM ( $3 \mu \mathrm{mol} / 1)$ for indicated periods were subjected to western blot analysis of GRP78 expression. (B) Breast cancer cells were relatively resistant to ER stress-induced apoptosis. MCF-7, MDA-MB-231, MCF-10A and WI-38 cells with or without treatment with TM at indicated concentrations for $48 \mathrm{~h}$ were subjected to measurement of apoptosis by the propidium idide (PI) method using flow cytometry (FCM). "P $<0.5$ for TM-induced apoptotic cells in MCF-10A compared to MCF-7 and MDA-MB-231 cells for indicated concentrations, respectively; ${ }^{* *} \mathrm{P}<0.01$ for the same compared with the extreme concentration $(12 \mu \mathrm{mol} / \mathrm{l}) .{ }^{*} \mathrm{P}<0.5$ for WI-38 compared to MCF-7 and MDA-MB-231 cells, respectively; ${ }^{\# \#} \mathrm{P}<0.01$ for the same compared with the extreme concentration $(12 \mu \mathrm{mol} / \mathrm{l})$.

A

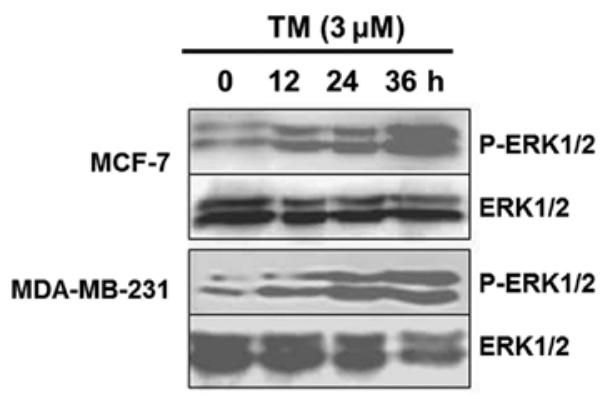

B

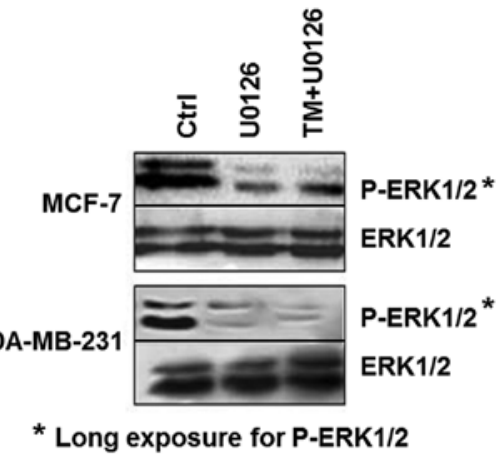

C

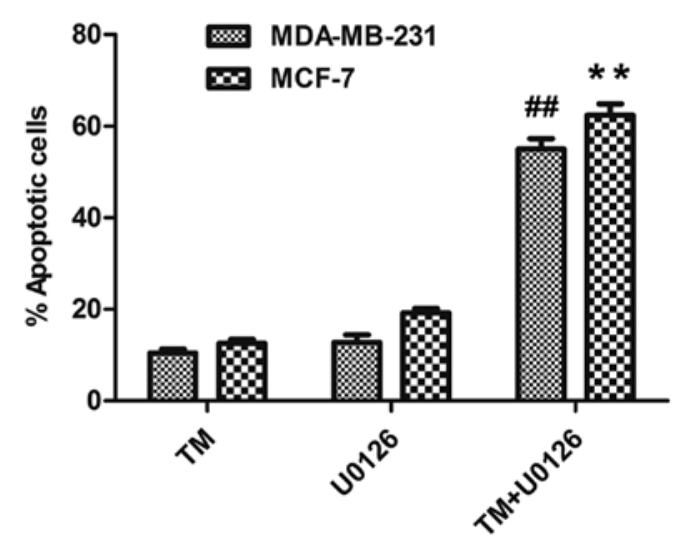

Figure 3. Inhibition of MEK sensitized breast cancer cells to ER stress-induced apoptosis. (A) Tunicamycin (TM) induced further activation of ERK1/2 in breast cancer cells. Whole-cell lysates from MCF-7 and MDA-MB-231 cells with or without treatment with TM (3 $\mu$ mol/1) for indicated periods were subjected to western blot analysis of ERK1/2 and P-ERK1/2 expression. (B) U0126 blocked activation of ERK1/2, with or without TM. Whole-cell lysates from MDA-MB-231 and SK-BR-3 cells with TM $(3 \mu \mathrm{mol} / \mathrm{l})$, U0126 $(20 \mu \mathrm{mol} / \mathrm{l})$ or combination of TM and U0126 for $24 \mathrm{~h}$ were subjected to western blot analysis of ERK1/2 and P-ERK1/2 expression. (C) U0126 sensitized breast cancer cells to ER stress-induced apoptosis. MCF-7 and MDA-MB-231 cells with TM ( $3 \mu \mathrm{mol} / 1)$, U0126 (20 $\mu \mathrm{mol} / \mathrm{l})$ or combination of TM and U0126 for $48 \mathrm{~h}$ were subjected to measurement of apoptosis by the PI method using FCM. ${ }^{* *} \mathrm{P}<0.01$ for the combination of TM and U0126 compared to TM and U0126 alone, respectively, in MCF-7 cells. ${ }^{\# \#} \mathrm{P}<0.01$ for the combination of TM and U0126 compared to TM and U0126 alone, respectively, in MDA-MB-231 cells.

exposure to U0126, a special MEK inhibitor, resulted in reduction in the levels of GRP78 expression and attenuated its induction by TM in both MCF-7 and MDA-MB-231 cells. To examine if inhibition of MEK/ERK blocked transcription of GRP78, we monitored mRNA levels of GRP78 by RT-qPCR in MCF-7 and MDA-MB-231 cells treated with U0126 $1 \mathrm{~h}$ before TM treatment. As shown in Fig. 4B, GRP78 mRNA levels in cells treated with TM in the presence of U0126 were markedly lower than those in cells treated with TM alone $(\mathrm{P}<0.01$, Student's t-test).

GRP78 knockdown by siRNA sensitizes breast cancer cells to ER stress-induced apoptosis. To further study if sensitization of breast cancer cells to ER stress-induced apoptosis was due 

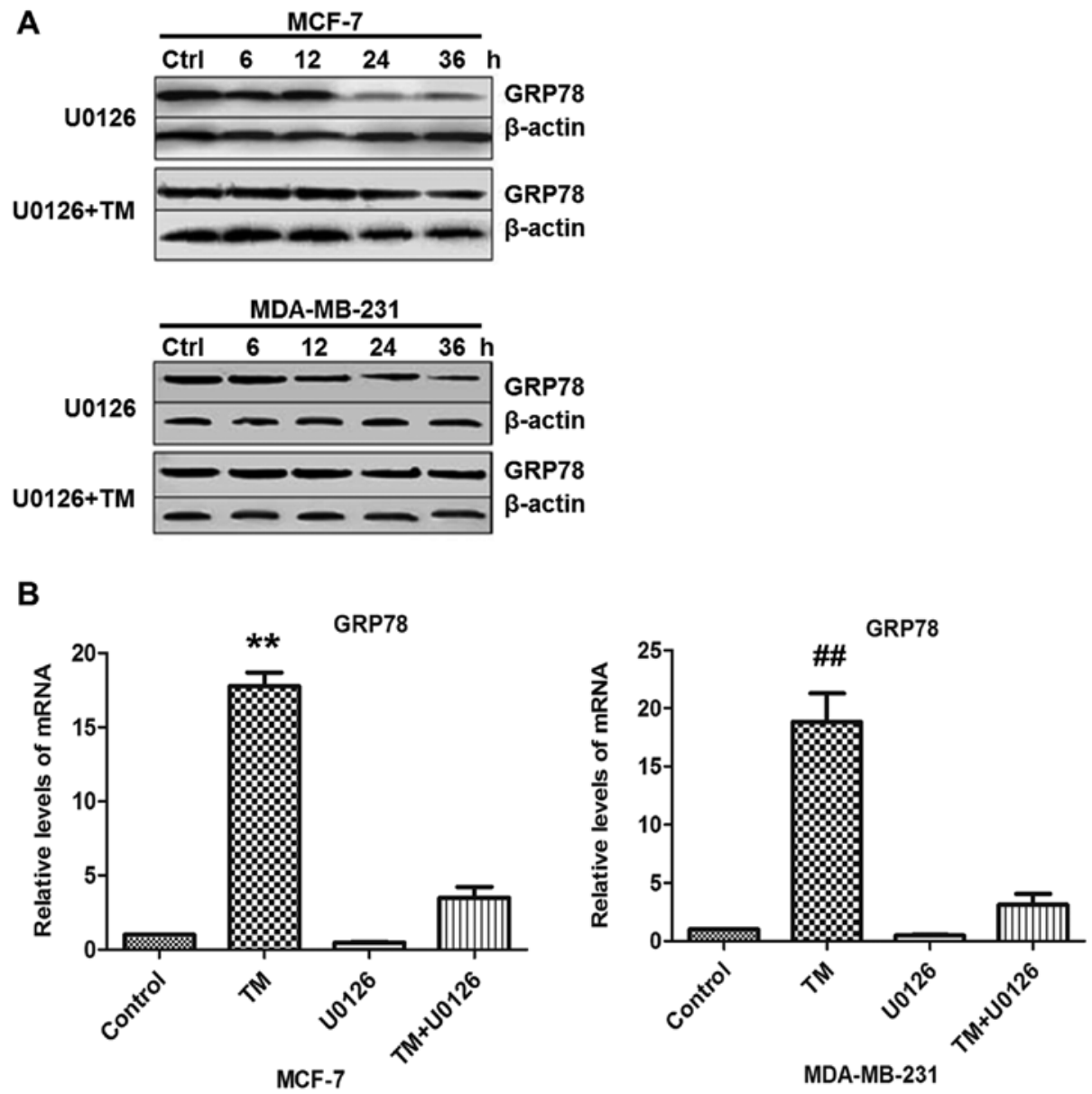

Figure 4. Inhibition of MEK/ERK decreased GRP78 expression. (A) U0126 inhibited GRP78 expression. Whole-cell lysates from MCF-7 and MDA-MB-231 cells treated with U0126 (20 $\mu \mathrm{mol} / 1)$ or combination with TM $(3 \mu \mathrm{mol} / \mathrm{l})$ for indicated periods were subjected to western blot analysis of GRP78 expression. (B) GRP78 mRNA expression were analyzed in MCF-7 and MDA-MB-231 cells treated with U0126 (20 $\mu$ mol/1) with or without the addition of TM ( $3 \mu \mathrm{mol} / 1)$ for indicated periods by RT-qPCR. * $\mathrm{P}<0.01$ for TM-induced GRP78 mRNA expression compared to TM combination with U0126 in MCF-7 cells. ${ }^{\# \prime} \mathrm{P}<0.01$ for the same compared with MDA-MB-231 cells.

to reduced GRP78 expression, we used siRNA knock-down of GRP78, then observed ER stress-induced apoptosis by TM in MCF-7 and MDA-MB-231 cells. As shown in Fig. 5A exposure to GRP78 siRNA resulted in blockage of GRP78 expression in both MCF-7 and MDA-MB-231 cells, while control siRNA had no effect on the levels of GRP78 expression, compared to the negative group. As shown in Fig. 5B, inhibition of GRP78 by siRNA resulted in substantial increases in sensitivity of breast cancer cells to apoptosis induced by TM in the absence of U0126 ( $\mathrm{P}<0.01$, Student's t-test). MCF-7 and MDA-MB-231 cells were treated with U0126 $1 \mathrm{~h}$ before TM treatment, along with GRP78 knockdown by siRNA, which resulted in more significant increases in sensitivity of cells to apoptosis $(\mathrm{P}<0.01$, Student's t-test). However, inhibition of GRP78 by siRNA only failed to induce significant apoptosis in both MCF-7 and MDA-MB-231 cells.

Caspase- 4 activity was monitored to examine whether sensitization of breast cancer cells to TM-induced apoptosis by inhibition of MEK/ERK is caspase-dependent. As shown in Fig. 5C, tunicamycin (TM) or U0126 alone did not constitutively activate caspase-4, whereas, TM along with U0126 markedly activated it in that caspase- 4 activity was increased by 2 -fold $(\mathrm{P}<0.05$, Student's t-test). Furthermore, knockdown of GRP78 by siRNA and TM caused an increase of 2 -fold caspase- 4 activity $(\mathrm{P}<0.05$, Student's t-test). Altogether GRP78 siRNA with TM in the presence of U0126, caspase-4 was 4-fold increased in both MCF-7 and MDA-MB-231 cells $(\mathrm{P}<0.01$, Student's t-test).

\section{Discussion}

Resistance to chemotherapy is a major obstacle to improving therapeutic effects of breast cancer. The study of resistance mechanisms is becoming a new strategy of overcoming various cancers. The above results suggest that there are two apoptotic resistance mechanisms in breast cancer which are constitutively activation of MEK/ERK pathway and stress induction of the UPR. GRP78 was found to be expressed at relatively high levels in cultured breast cancer cells and tissue, but were at low levels in cultured breast normal epithelial cells and normal tissue, which suggest that the UPR may have tumor-specific selectivity. The strongly positive GRP78 expression suggests that the UPR is acutely activated in breast cancer and targeted therapy against breast cancer via surface GRP78 may antagonize the cyto-protective UPR.

TM, an ER stress inducer, markedly upregulated GRP78 expression and in $24 \mathrm{~h}$ the upregulation peaked. Furthermore, cultured breast cancer cells did not undergo significant 
A

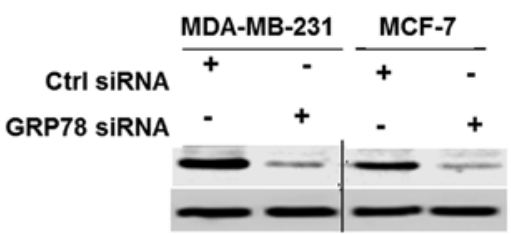

B

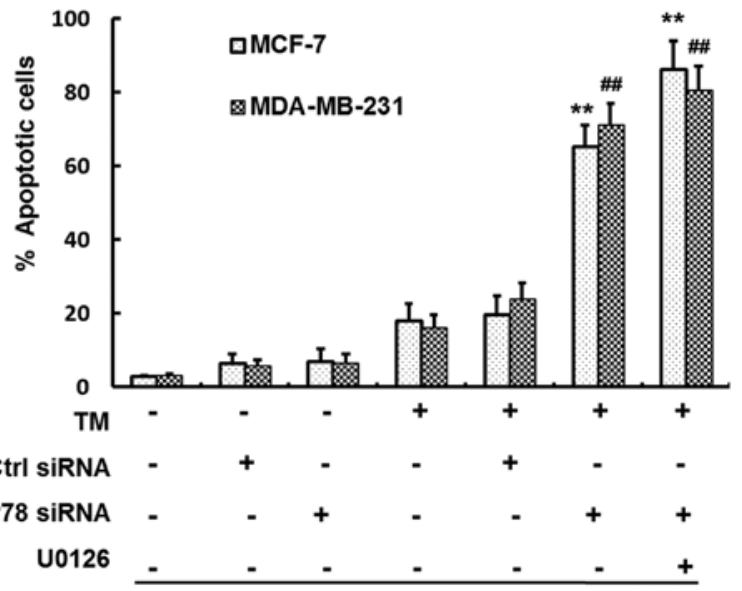

C

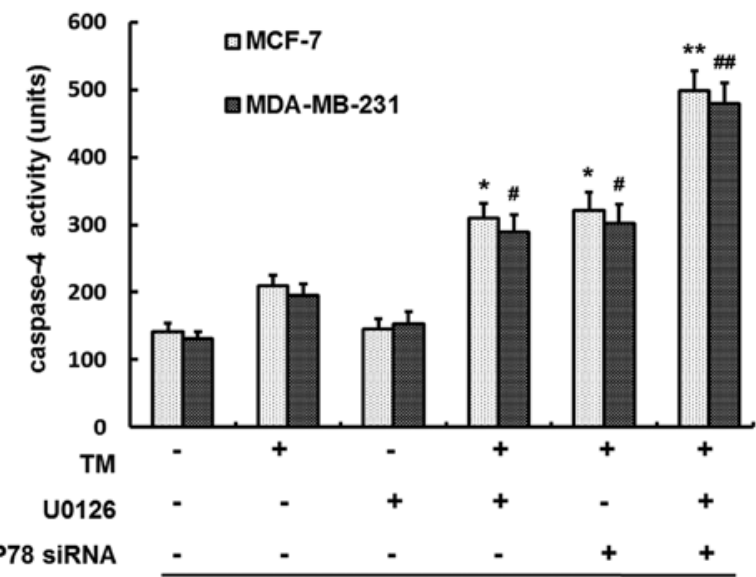

Figure 5. GRP78 knockdown by siRNA sensitizes breast cancer cells to TM-induced apoptosis and the sensitization is at least in part caspase- 4 dependent. (A) Efficiency of knockdown of GRP78 by siRNA. Whole-cell lysates from MCF-7 and MDA-MB-231 cells with control siRNA or GRP78 siRNA transfection for $24 \mathrm{~h}$ were subjected to western blot analysis of GRP78 expression. (B) siRNA knock-down of GRP78 expression sensitized breast cancer cells to ER stress-induced apoptosis. MCF-7 and MDA-MB-231 cells were transfected with the control or GRP78 siRNA, 24 h later, the cells were treated with TM $(3 \mu \mathrm{mol} / \mathrm{l})$ for $48 \mathrm{~h}$ or $\mathrm{U} 0126$ for $1 \mathrm{~h}$ before the addition of TM for further $48 \mathrm{~h}$. Apoptosis was measured by the PI method using FCM. ${ }^{* *} \mathrm{P}<0.01$ for the two and three combinations of TM, GRP78 siRNA and U0126 compared to TM, GRP78 siRNA and U0126 alone, respectively, in MCF-7 cells. ${ }^{\# \#} \mathrm{P}<0.01$ for the same compare in MDA-MB-231 cells. (C) Sensitization of breast cancer cells to TM-induced apoptosis was caspase-4 dependent, at least in part. Whole-cell lysates from MCF-7 and MDA-MB-231 cells treated with U0126 (20 $\mu \mathrm{mol} / 1)$, TM $(3 \mu \mathrm{mol} / 1)$, GRP78 siRNA and their combinations of two or three for $24 \mathrm{~h}$ were subjected to caspase- 4 activity assay. ${ }^{*} \mathrm{P}<0.01$ for the combination of TM, GRP78 siRNA or U0126 compared to TM, GRP78 siRNA and U0126 alone, respectively, in MCF-7 cells; ${ }^{\text {}} \mathrm{P}<0.01$ for the same compared with MDA-MB-231 cells. ${ }^{* *} \mathrm{P}<0.01$ for the three combination of TM, GRP78 siRNA and U0126 compared to TM, GRP78 siRNA and U0126 alone, respectively, in MCF-7 cells. ${ }^{\# \#} \mathrm{P}<0.01$ for the same compared with MDA-MB-231 cells.

apoptosis when submitted to extreme degrees of ER stress induced by TM, but the same treatment induced significant apoptosis in normal epithelial cells. Obviously, TM aggravated ER stress in cancer cells and further activated UPR to protect cells against damage from stress, thus causing ER stress-induced apoptosis. GRP78 expression may serve as a biomarker for activation of the UPR and play anti-apoptotic properties of the UPR in breast cancer. This could explain why TM did not induce significant apoptosis in breast cancer cells, differentiating from normal cells. It is indicated that the cyto-protective UPR is the base of tumor resistance and GRP78 plays a central role in the process.

The MAPK pathway is known to be activated in a broad spectrum of human tumors, including human colorectal (25) and gastric cancer (26) and melanoma (27). We observed that activation of the MEK/ERK pathway was relatively weak in breast cancer cells, yet TM induced constitutive activation of the pathway as evidenced by increased phosphorylation (activation) form of ERK1/2 (P-ERK1/2). The MEK inhibitor was found to downregulate GRP78 expression and block activation of the UPR induced by TM. This indicates that activation of the MEK/ERK pathway plays an important role in upregulation of GRP78 by ER stress. In light of these results, we consider that the MEK inhibitor functions upstream of the activation of UPR and inhibiting the activation of survival pathway MEK/ERK blocks the cyto-protective role of the UPR. It was confirmed by ER stress-induced apoptosis, which is readily triggered when the MEK/ERK pathway is inhibited by the MEK inhibitor U0126. We also found that the MEK inhibitor alone did not cause appreciable apoptotic cell death in breast cancer cells. Therefore the MEK/ERK pathway inhibition would be responsible for the increasing sensitivity of ER stress-induced apoptosis. Furthermore, the ER chaperon GRP78 seems to be a target of the MEK/ERK pathway responsible for the inhibition of ER stress-induced apoptosis. We consider that inhibiting GRP78 expression may enhance ER stress-induced apoptosis.

GRP78 expression is primarily regulated at the transcriptional level, mediated by multiple copies of the ER stress response element within the GRP78 promoter region (28). We showed by RT-qPCR that GRP78 mRNA levels were decreased by inhibition of MEK/ERK in the presence or absence of $\mathrm{TM}$, indicating that the MEK/ERK pathway may participate in regulation of GRP78 transcription. GRP78 is induced in a wide variety of cancer cells and cancer biopsy tissue. The identification of the transcription factors interacting with the ER stress response element leads to the discovery of multiple pathways whereby mammalian cells can sense ER stress and trigger the transcription of GRP78 (29). We utilized GRP78 knockdown by siRNA resulting in significant increases in sensitivity of breast cancer cells to TM-induced apoptosis, but GRP78 siRNA alone, without TM induction, resulted in only slight increase in apoptosis. This suggests that GRP78 seems to be a target of ER stress-induced apoptosis which it appears anti-apoptotic only when ER stress is induced.

We also found that sensitization of breast cancer cells to TM-induced apoptosis by inhibition of MEK/ERK was caspase-4 dependent, in that when TM induced ER stress, caspase-4 activity was lower, yet MEK/ERK inhibition and GRP78 knockdown increased levels of caspase-4 activity. Caspase-4 is an apical caspase in induction of apoptosis by TM in the presence of U0126, activation of caspase-4 necessarily switched on caspase cascade responses to apoptosis. Moreover, inhibition of GRP78 by siRNA 
enhanced activation of caspase- 4 induced by TM. These results indicate that GRP78 may participate in controlling the activation of caspase- 4 in breast cancer cells.

In summary, GRP78 knockdown resulted in significant increases in sensitivity of cells to apoptosis by TM. Inhibition of MEK/ERK may sensitize breast cancer cells to TM-induced apoptosis. The results indicate that it may be an effective strategy against breast cancer to combine agents that induce ER stress with those inhibiting the MEK/ERK pathway. However, normal cells WI-38 and MCF-10A seem to be relatively sensitive to TM-induced apoptosis, indicating that careful evaluation of clinically relevant ER stress-inducing agents in combination with inhibitors targeting the MEK/ERK pathway is required before in vivo investigations being carried out. Of interest, GRP78 is found to be expressed at relatively high levels in cultured breast cancer cells and breast cancer tissues, but is hardly detectable in WI-38, MCF-10A and breast fibroadenoma. This suggests that targeting GRP78 may have tumor-specific selectivity and thus being useful in treatment of breast cancer, this role is due, at least partly, to activation of caspase-4. GRP78 may be a potential target against breast cancer, and small molecular inhibitor targeting GRP78 in combination with those inducing ER stress, is a promising chemotherapeutical plan against breast cancer.

\section{Acknowledgements}

This study was supported by grants from the National Natural Science Foundation of China (31401094).

\section{References}

1. Pluquet O, Pourtier A and Abbadie C: The unfolded protein response and cellular senescence. A review in the theme: Cellular mechanisms of endoplasmic reticulum stress signaling in health and disease. Am J Physiol Cell Physiol 308: C415-C425, 2015.

2. Manié SN, Lebeau J and Chevet E: Cellular mechanisms of endoplasmic reticulum stress signaling in health and disease. 3. Orchestrating the unfolded protein response in oncogenesis: An update. Am J Physiol Cell Physiol 307: C901-C907, 2014.

3. Bian Q, Fernandes AF, Taylor A, Wu M, Pereira P and Shang F: Expression of K6W-ubiquitin in lens epithelial cells leads to upregulation of a broad spectrum of molecular chaperones. Mol Vis 14: 403-412, 2008.

4. Shoulders MD, Ryno LM, Genereux JC, Moresco JJ, Tu PG, Wu C, Yates JR III, Su AI, Kelly JW and Wiseman RL: Stressindependent activation of XBP1s and/or ATF6 reveals three functionally diverse ER proteostasis environments. Cell Rep 3: 1279-1292, 2013.

5. Ni M and Lee AS; Ni MandLee AS: ER chaperones in mammalian development and human diseases. FEBS Lett 581: 3641-3651, 2007.

6. Jaronen M, Goldsteins G and Koistinaho J: ER stress and unfolded protein response in amyotrophic lateral sclerosis-a controversial role of protein disulphide isomerase. Front Cell Neurosci 8: 402, 2014.

7. Rainbolt TK, Saunders JM and Wiseman RL: Stress-responsive regulation of mitochondria through the ER unfolded protein response. Trends Endocrinol Metab 25: 528-537, 2014.

8. Wang M, Wey S, Zhang Y, Ye R and Lee AS: Role of the unfolded protein response regulator $\mathrm{GRP} 78 / \mathrm{BiP}$ in development, cancer, and neurological disorders. Antioxid Redox Signal 11: 2307-2316, 2009.

9. Matsuo K, Gray MJ, Yang DY, Srivastava SA, Tripathi PB, Sonoda LA, Yoo EJ, Dubeau L, Lee AS and Lin YG: The endoplasmic reticulum stress marker, glucose-regulated protein-78 (GRP78) in visceral adipocytes predicts endometrial cancer progression and patient survival. Gynecol Oncol 128: 552-559, 2013.

10. Schröder M and Kaufman RJ: ER stress and the unfolded protein response. Mutat Res 569: 29-63, 2005.
11. Wang C, Jiang K, Gao D, Kang X, Sun C, Zhang Q, Li Y, Sun L, Zhang S, Guo K, et al: Clusterin protects hepatocellular carcinoma cells from endoplasmic reticulum stress induced apoptosis through GRP78. PLoS One 8: e55981, 2013.

12. Pootrakul L, Datar RH, Shi SR, Cai J, Hawes D, Groshen SG, Lee AS and Cote RJ: Expression of stress response protein Grp78 is associated with the development of castration-resistant prostate cancer. Clin Cancer Res 12: 5987-5993, 2006.

13. Mhaidat NM, Wang Y, Kiejda KA, Zhang XD and Hersey P: Docetaxel-induced apoptosis in melanoma cells is dependent on activation of caspase-2. Mol Cancer Ther 6: 752-761, 2007.

14. Choudhury S, Bhootada Y, Gorbatyuk O and Gorbatyuk M: Caspase-7 ablation modulates UPR, reprograms TRAF2-JNK apoptosis and protects T17M rhodopsin mice from severe retinal degeneration. Cell Death Dis 4: e528, 2013.

15. Estornes Y, Aguileta MA, Dubuisson C, De Keyser J, Goossens V, Kersse K, Samali A, Vandenabeele P and Bertrand MJ: RIPK1 promotes death receptor-independent caspase-8-mediated apoptosis under unresolved ER stress conditions. Cell Death Dis 6: e1798, 2015.

16. Banerjee C, Singh A, Das TK, Raman R, Shrivastava A and Mazumder S: Ameliorating ER-stress attenuates Aeromonas hydrophila-induced mitochondrial dysfunctioning and caspase mediated HKM apoptosis in Clarias batrachus. Sci Rep 4: 5820, 2014.

17. Sandow JJ, Dorstyn L, O'Reilly LA, Tailler M, Kumar S, Strasser A and Ekert PG: ER stress does not cause upregulation and activation of caspase-2 to initiate apoptosis. Cell Death Differ 21: 475-480, 2014.

18. Binet F, Chiasson S and Girard D: Evidence that endoplasmic reticulum (ER) stress and caspase-4 activation occur in human neutrophils. Biochem Biophys Res Commun 391: 18-23, 2010.

19. Matsuzaki S, Hiratsuka T, Kuwahara R, Katayama T and Tohyama M: Caspase- 4 is partially cleaved by calpain via the impairment of $\mathrm{Ca}^{2+}$ homeostasis under the ER stress. Neurochem Int 56: 352-356, 2010.

20. Adachi T, Teramachi M, Yasuda H, Kamiya T and Hara H: Contribution of $\mathrm{p} 38 \mathrm{MAPK}, \mathrm{NF}-\mathrm{\kappa B}$ and glucocorticoid signaling pathways to ER stress-induced increase in retinal endothelial permeability. Arch Biochem Biophys 520: 30-35, 2012.

21. Feng R, Zhai WL, Yang HY, Jin H and Zhang QX: Induction of ER stress protects gastric cancer cells against apoptosis induced by cisplatin and doxorubicin through activation of p38 MAPK. Biochem Biophys Res Commun 406: 299-304, 2011.

22. Lin ML, Chen SS, Huang RY, Lu YC, Liao YR, Reddy MV, Lee CC and Wu TS: Suppression of PI3K/Akt signaling by synthetic bichalcone analog TSWU-CD4 induces ER stressand Bax/Bak-mediated apoptosis of cancer cells. Apoptosis 19: 1637-1653, 2014

23. Wang Z, Zhang H, Xu X, Shi H, Yu X, Wang X, Yan Y, Fu X, $\mathrm{Hu} \mathrm{H}, \mathrm{Li} \mathrm{X}$, et al: bFGF inhibits ER stress induced by ischemic oxidative injury via activation of the PI3K/Akt and ERK1/2 pathways. Toxicol Lett 212: 137-146, 2012.

24. Lee AS: The ER chaperone and signaling regulator GRP78/BiP as a monitor of endoplasmic reticulum stress. Methods 35: 373-381, 2005.

25. Mhaidat NM, Alali FQ, Matalqah SM, Matalka II, Jaradat SA, Al-Sawalha NA and Thorne RF: Inhibition of MEK sensitizes paclitaxel-induced apoptosis of human colorectal cancer cells by downregulation of GRP78. Anticancer Drugs 20: 601-606, 2009.

26. Kang W, Tong JH, Chan AW, Lee TL, Lung RW, Leung PP, So KK, Wu K, Fan D, Yu J, et al: Yes-associated protein 1 exhibits oncogenic property in gastric cancer and its nuclear accumulation associates with poor prognosis. Clin Cancer Res 17: 2130-2139, 2011.

27. Oh YT, Deng J, Yue P, Owonikoko TK, Khuri FR and Sun SY: Khuri FR and Sun SY: Inhibition of B-Raf/MEK/ERK signaling suppresses DR5 expression and impairs response of cancer cells to DR5-mediated apoptosis and T cell-induced killing. Oncogene: Apr 13, 2015 (Epub ahead of print). doi: 10.1038/onc.2015.97.

28. Tang J, Guo YS, Zhang Y, Yu XL, Li L, Huang W, Li Y, Chen B, Jiang JL and Chen ZN: CD147 induces UPR to inhibit apoptosis and chemosensitivity by increasing the transcription of Bip in hepatocellular carcinoma. Cell Death Differ 19: 1779-1790, 2012.

29. Li J and Lee AS: Stress induction of GRP78/BiP and its role in cancer. Curr Mol Med 6: 45-54, 2006. 